\title{
A Scoping Review of Interventions Delivered by Peers to Support the Resettlement Process of Refugees and Asylum Seekers
}

\author{
Daryl Mahon
}

Citation: Mahon, D. A Scoping Review of Interventions Delivered by Peers to Support the Resettlement Process of Refugees and Asylum Seekers. Trauma Care 2022, 2, 51-62. https://doi.org/10.3390/ traumacare2010005

Received: 29 December 2021 Accepted: 21 February 2022 Published: 25 February 2022

Publisher's Note: MDPI stays neutral with regard to jurisdictional claims in published maps and institutional affiliations.

Copyright: (c) 2022 by the author. Licensee MDPI, Basel, Switzerland. This article is an open access article distributed under the terms and conditions of the Creative Commons Attribution (CC BY) license (https:// creativecommons.org/licenses/by/ $4.0 /)$.
Genio, D01 Dublin, Ireland; daryl.mahon@genio.ie

\begin{abstract}
Background: Refugees and asylum seekers face many social and psychological challenges on their journey to resettlement in host countries. Interventions and programmes designed to assist in these challenges are necessary. The aim of this scoping review is to conduct a systematic search of the literature as it pertains to interventions delivered by peers to refugees and asylum seekers during the resettlement process. Methods: A PRISMA-compliant scoping review was conducted. Four databases, Scopus, Embase, Ebsco, and ScienceDirect were searched for peer-reviewed articles published in English from 2000-2021. Studies were included if they reported on interventions, outcomes or the training received by adult peers to support refugees and asylum seekers during the resettlement process. Results: Of an initial 639 journal articles retrieved, 14 met the inclusion criteria for this review. Most included studies were conducted in Western high-income countries, except for one. Studies were heterogeneous in terms of the nationalities of peers and those receiving peer interventions; the outcomes reported on; the content of interventions; and the methodologies used. Conclusions: Findings suggest that peer interventions seem to be effective in addressing many of the challenges faced by refugees and asylum seekers. Community integration, acculturation and psychological distress are some of the key benefits. When such interventions are co-produced in participatory research involving refugees, asylum seekers, and the civil society organisations that support this population, they are naturally culturally responsive and can therefore address issues relative to different ethnic needs during the resettlement process.
\end{abstract}

Keywords: refugee; asylum seeker; resettlement; scoping review; psychological distress

\section{Introduction}

According to the World Migration Report [1], there is an estimated 281 million international migrants across the globe, equating to $3.6 \%$ of the population. In the last two decades over 65 million people have been displaced globally, with over 23 million individuals emerging from high-conflict countries such as Syria, Iraq, and South Sudan [2] Moreover, the United Nations High Commissioner for Refugees (UNHCR) reports that there are currently more than 30 million refugees and asylum seekers resettled in highincome countries [3]. Given the traumatic nature of these displacements, in addition to pre and post displacement stressors, many refugees and asylum seekers have elevated levels of psychological distress and social needs. Systematic reviews and meta-analyses demonstrate differential prevalence across disorders (3-88\%) for this population [4-8].

Systematic reviews and meta-analyses illustrate that refugees and asylum seekers have a wider set of systemic post-displacement psychosocial needs, which can exacerbate quality of life impairment and impact healthcare outcomes [9-11]. Some of these post-displacement needs include issues with communicating due to language/cultural barriers [12,13]. Exploring post displacement needs under ecological/social determinants of mental health framework has become more popular in the literature [14-17]. For example, a lack of adequate housing, barriers to employment and income generation, and loss of important social roles and social connections including family ties, are all linked to psychological 
distress during the resettlement, acculturation, and integration process $[9,10,18]$. As such, interventions that move beyond the individual psychopathology and have a social element are needed when working with this population, and these supports must be culturally responsive $[19,20]$.

Although there has been a proliferation of models to work with such issues across general populations in Western societies, a more nuanced approach that considers multicultural identities is needed when working with refugees and asylum seekers [21,22]. Research supports the presupposition that culture needs to be considered; the literature demonstrates small to medium effects when cultural adaptations are implemented by professionals across psychological and preventative interventions [19,23-25]. However, considering the heterogeneity within and between refugee and asylum populations, training professionals to meet these disparate cultural needs may be problematic. Riggs [26] informs us that "there may not be one 'model' of best practice but a suite of strategies that are flexible and adaptable and are reflective of the clients cultures, languages, existing social groups and resources of local service providers both mainstream and culturally-specific". Indeed, a recent scoping review on cultural competency in organisations working with refugees suggests that there is a lack of voice and participation by refugees and asylum seekers [20]. One method that may mitigate against a possible lack of cultural competency at the organisational level, and on the burden of training professionals in such approaches, while also encouraging more participation by refugees and asylum seekers, may be to use peers to deliver resettlement support.

\section{Peer Support}

Peer support has garnered increasing attention and support in recent decades, especially in the mental health space where lived experience and survivor voice is becoming more prevalent [27]. While there has been some disagreement as to the outcomes associated with peer support, studies have demonstrated a host of psychosocial and engagement outcomes [27-31].

In a review of general social service interventions offered to refuges and asylum seekers during resettlement, Shaw and Funk [32] identified three support programmes delivered by peers. Overall, the literature exposes a relative lack of research on peer support programmes delivered by refugees or asylum seekers internationally. This was also noted in other studies $[15,18]$. As such, a systematic scoping review is warranted as one way to identify and map the state of the extant knowledge in this arena.

\section{Materials and Methods}

A systematic scoping review of the published literature on peer support with refugees and asylum seekers during the resettlement process was conducted. The scoping review approach has been used very recently across differential studies with refugee and asylum seeker populations. For example,Burns et al. [33] used this approach to identify issues around health with migrants in the United Kingdom. In a similar study, Villarroel et al. [34] used a scoping review to explore health factors with refuges in Ireland. Furthermore, other studies used the approach to establish the extent of trust in resettlement settings [35]; to examine cultural competency in refugee service delivery [20]; to explore community-based healthcare best practices with refugees [22]; use of interpreters in mental health setting with refugees [36]; and to establish the extent of resettlement social supports [37].

This systematic scoping review is informed by The Preferred Reporting Items for Systematic Reviews and Meta-Analysis (PRISMA) standard reporting guidelines [38]. Arksey and O'Malley's five-stage scoping review framework was employed [39]. The review was conducted in the following steps, identifying the essential research question; identifying relevant studies; study selection; charting the data; collecting, summarising, and reporting the results. As part of the reporting process for this scoping review, the Preferred Reporting Items for Systematic Reviews and Meta-Analysis extension for scoping reviews (PRISMA-ScR) checklist is used for transparency and reproducibility purposes [40]. 


\subsection{Stage 1. Identifying the Essential Research Question}

The scoping review framework requires a broad and well-articulated research question, which clearly outlines the concepts, outcomes, and population of interest [39,41]. Thus, the following broad scoping question informed by the PICO protocol [42] was used to develop the research question. How does the peer-reviewed literature describe interventions delivered by peers to refugees and asylum seekers during resettlement? The following sub-questions will be reported on also.

1. What type of research methodologies are used in research of peer interventions during the resettlement process with refuges and asylum seekers?

2. What outcomes are reported on in the peer literature

3. What type of training regimes are provided to peers delivering interventions?

\subsection{Stage 2. Identifying Relevant Studies}

The scoping review literature suggests using several literature resources to build a comprehensive picture of the research as it pertains to the research question [39]. Database searches in Web of Science, Scopus, Embase, and Ebsco were supplemented with searches of reference lists of identified papers, and a hand search of three electronic journals identified as relevant to the research question. The following words were used to search databases in Scopus and then also used in the three other databases: Peer* AND Refugee* OR Asylum* AND Resettlement* and NOT Children* OR Adolescent*. In addition, emails were sent to national and international organisations working in the refugee and asylum seeker practice and policy space asking if they were aware of any relevant studies.

\subsection{Stage 3. Study Selection}

Inclusion and exclusion were initially developed at the outset of the study and refined based on an iterative process after initial searches [39]. This criterion was chosen as it provides the best opportunity to address the research question/s. Due to constraints on time and resources it was only feasible to include studies conducted in English. Only methodologies that provided primary data on the delivery of the interventions or training from 2000-2021 were included. The rationale for these inclusion dates is based on the number of people displaced globally in the last two decades (65 million), as outlined in the introduction to this paper.

In total, 620 articles were sourced from databases, with 19 additional articles identified through citation searching as the study progressed $(n=639)$. All articles were downloaded into the reference management software Mendeley for appraising. The author screened all titles and abstracts in Mendeley, excluding those that failed to meet the inclusion criteria. A deadline for any new articles to be identified and added to the review was set as 10 November 2021. A total of 14 articles met inclusion criteria for this scoping review. The Preferred Reporting Items for Systematic Reviews and Meta-Analysis extension for scoping reviews (PRISMA) flowchart outlines the process involved in this study selection (Figure 1).

Depicts the flow of information through the different phases of the scoping review and the rationale for inclusion/exclusion.

\subsection{Stage 5. Collecting, Summarising, and Reporting the Results}

For reporting the results, a narrative summary based on thematic analysis [43] is provided. Relevant themes related to the research question/s were developed based on data extracted from the charting form. Codes were assigned in Microsoft Word and then assimilated into broader abstract themes, which are discussed in this section. The data charting form was developed by the author, and it illustrates the characteristics used to extract data from individual studies. These heading were then utilised to inform the first coding phase. See Table S1 Supplementary Material. 


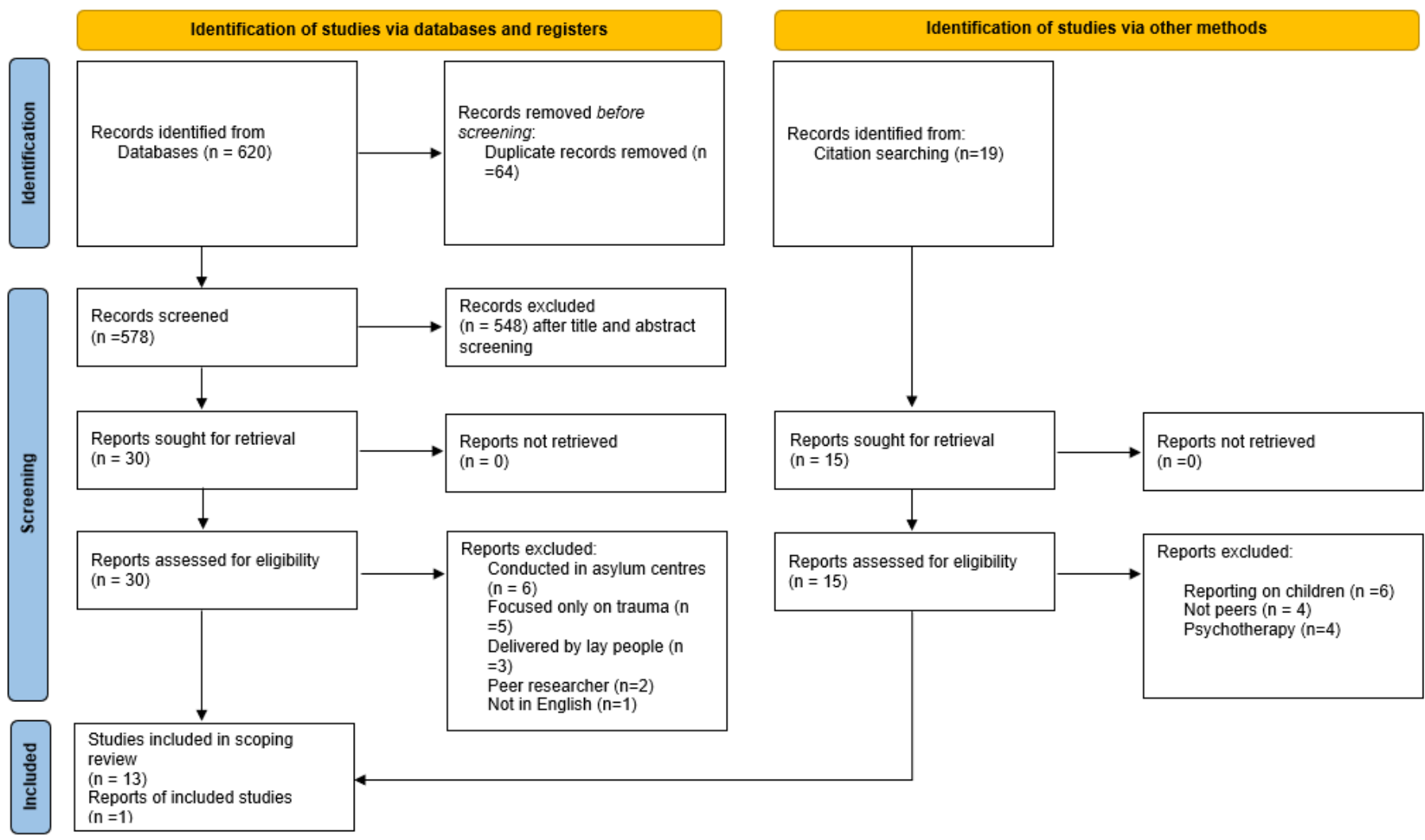

Figure 1. PRISMA Flowchart adaptation as outlined by Page et al. [38].

\section{Results}

Most studies $(\mathrm{N}=13)$ were carried out in high income Western societies, America $(\mathrm{N}=4)$, Canada $(\mathrm{N}=1)$, Australia $(\mathrm{N}=2)$, Spain $(\mathrm{N}=2)$, Sweden $(\mathrm{N}=1)$, Austria $(\mathrm{N}=2)$, Netherlands $(\mathrm{N}=1)$, Germany $(\mathrm{N}=1)$, Italy $(\mathrm{N}=1)$, England $(\mathrm{N}=1)$, Scotland $(\mathrm{N}=1)$, and Finland $(\mathrm{N}=1)$. One study $(\mathrm{N}=1)$ was conducted in a non-high-income country, Uganda. For the studies that reported on participants nationality $(\mathrm{N}=14)$, Baltic states were reported in $(\mathrm{N}=1)$, Chechnya $(\mathrm{N}=1)$, Latino $(\mathrm{N}=1)$, Africa $(\mathrm{N}=6)$, Middle East $(\mathrm{N}=4)$, Southeast Asia $(\mathrm{N}=4)$, Afghanistan $(\mathrm{N}=2)$, and Pakistan $(\mathrm{N}=1)$. Sample sizes were reported in all studies with a range of $(\mathrm{N}=9-\mathrm{N}=613)$. Methodologies were reported in all studies $(\mathrm{N}=14)$; qualitative studies were used in $(\mathrm{N}=6)$, of which $(\mathrm{N}=2)$ were evaluations, $(\mathrm{N}=4)$ were quantitative, of which three $(\mathrm{N}=3)$ were a randomised control trial (RCT), with active controls, and mixed methods were reported in $(\mathrm{N}=5)$. A co-production/participatory approach was used to inform the design of the interventions and/or access participants in $(\mathrm{N}=8)$, largely this was achieved through partnering with existing structures such as NGOs or community leaders. $(\mathrm{N}=3)$ studies made explicit reference to making groups/interventions more homogeneous based on cultural demographics. The type of training peers received was reported in $(\mathrm{N}=13)$, and pre-existing manuals/protocols for the interventions were available in $(\mathrm{N}=5)$.

\subsection{The Impact of Training on Peers and Their Roles}

The training provided to peers to prepare them for their respective roles was heterogeneous. Although this can make it more difficult for reporting on evidence synthesis, it may speak to the fact that it is an area with a dearth of research, and the differential cultural and resettlement needs of refugees and asylum seekers in each study.

In terms of the duration of training provided to peers, workshops ranged from $18 \mathrm{~h}$ [44] to $180 \mathrm{~h}$ [45] delivered by researchers or host organisations providing services to refugees in the community. While three studies had existing manuals [46-48], these studies were based on the amelioration of psychological distress. Likewise, the programme 
content was far from homogeneous. However, most peers received practical facilitation and communication skills training [49-54].

Several studies reported on methods used in the training to assist peers to understand and connect refugees and asylum seekers to further resources within the community [44,49-51,53,55].

Two studies described the qualitative experience peers had when it came to training or lack thereof. The supervisors and peers in the de Graaff et al. [46] study noted the importance of supervision for support, learning, and integrating the protocols. As this participant notes:

"Of course, we also had weekly supervision, so we talk about it. We are very clear and open about the difficulties we face, how we can better approach it, from how other colleagues dealt with it".

Interestingly, the one study that used a sample who had not received peer training prior to taking up their role noted the impact a lack of training had on both communication skills, and connecting refuges and asylum seekers to further resource:

"Interviewees said they need ongoing training around systems and resources, how to interact with colleagues and clients, and how to act and communicate with clients who may be upset, or frustrated. Caseworkers said they wanted opportunities for certification and professional training. Multiple interviewees talked about wanting qualifications beyond their experience". [56]

While this highlights the importance of training in these specific areas for peers, the optimal training regime in terms of content and duration remains elusive. One other issue to note is the impact that training had on peers' sense of self. In the Shaw [56] study, the lack of training may have left peers feeling ill prepared for some of the more challenging issues involved in their roles. In comparison, Paloma et al. [57] illustrate that peers in their study grew in resilience and feelings of empowerment as the training progressed, these issues would seem to be reflected in the three studies where peers received training and support based on existing manuals [46-48]. Overall, training seems to be integral for peers working with refugees and asylum seekers, while relying on experience alone has the potential to impact negatively on peers' wellbeing and feelings of effectiveness.

\subsection{Co-Producing Culturally Sensitive Peer Training Leads to Positive Outcomes}

Most of the studies that reported on a training regime included key stakeholders in a coproduced peer training, however the manualised protocols did not, although they adapted the intervention to be culturally responsive through peer delivery. Stakeholder involvement was achieved in various ways, such as directly consulting the peers on the type of content to be included, to partnering with key NGOs working within these areas, and consulting with community leaders from the identified communities. The type of discussion topics or content to be used in the peer training was developed in consultation with peers/recipients in [49,51,53-55]. In one study Abrahamson et al. [49], content was developed in conjunction with a theoretical model by using workshops to explore the narrative experience of the refugee process by the peers and integrating this with theoretical concepts in social health, psychology, and medical anthropology. In a second study, Steward et al. [53] used a preintervention process for refugees and asylum seekers to articulate their preferences for the type of supports they would like during the intervention. In doing so, interventions and content were developed to meet diverse needs, whilst also being culturally responsive.

Most of the studies $(\mathrm{N}=12)$ used the existing structures within the community, organisations, or community leaders to support the development of culturally responsive interventions. This was performed for two main reasons, to access participants and peers and to develop programmes as culturally sensitive. Community leaders were used in three studies [52-54] for accessing and choosing participants and designing culturally responsive content. Similarly, relevant organisations were involved as they had access to participants 
and could also inform the design of culturally responsive interventions [49,51,53-55]. Im and Rosenberg [51] elucidate what this process resembled in their study:

"For a culturally sensitive and effective intervention, the trained refugee leaders were actively involved in the development and adaptation process by providing inputs and feedback on the topics and the contents and adding culturally relevant examples and activities to the curriculum (ex. Bhutanese proverbs regarding health, chanting for opening and closure, etc.)".

While the content design and delivery were developed within a culturally sensitive coproduced format, the composition of peer interventions that were group based were also coproduced. The studies reported on the importance of having a homogeneous composition in terms of group membership, and this extended beyond ethnicity, with gender and even age often sought out $[47,49,52]$. The Steward et al. (2012, p. 525) [53] findings exemplify this:

"Participants appreciated that female and male refugees met separately and that the Somali and Sudanese refugees had separate groups. A few suggested that groups could be even more exclusive in terms of language and age. To illustrate, one Sudanese group comprised people who spoke different versions of Arabic making communication challenging. Agebased support interventions were suggested to enable people of the same age group to discuss similar issues affecting them".

In terms of what the intervention's peers were trained in, two models were based on training peers in peer support and then providing them with mobile phones with credit to practice offering support to each other in order to build social capital [52,54]. A third model [53] used a phone call as a follow up intervention for extra support by peers after groups to check in. Five interventions had existing established protocols/manuals predetermined prior to the study. The Club House Model [50]; Culture-Sensitive and Resource Oriented Peer (CROP); [45], ALMA (Amigas Latinas Motivando el Alma/Latina Friends Motivating the Soul) [44]; Self Help Plus [47,48] and Programme Management Plus [46]. The delivery and content of interventions was diverse, for example, Paloma et al. [57] reported including issues such as migratory mourning (e.g., social network, language, culture, and status) and identifying personal strengths and community resources to cope with these issues.

Instead, Steward et al. [53] used provision of information, affirmation, and emotional support in addition to accessibility (e.g., childcare and transportation). The range of information and supports delivered to refuges and asylum seekers was vast and highlights the importance of experiential knowledge supported by more formal content, including sources from the wider social system, as described by the Steward et al. [53]:

"Facilitators provided information on conflict management, financial counselling, addressing spousal conflicts, supporting children with schoolwork, dealing with discrimination, accessing services and seeking optimum employment. This information was derived from settlement agencies, government departments or the internet, in addition to peer facilitators' experiential knowledge".

Although the CROP intervention in the Renner et al. [45] study was primarily aimed at the amelioration of trauma in a culturally responsive way, the actual interventions delivered after the training could be conducted in a flexible manner decided by the peers. As such, the groups offered a mix of content and processes as illustrated in each gendered group where women discussed issues such as childcare, household affairs, or cooking, while men adhered to themes such as how to deal with the authorities in a proper way, how to obtain an Austrian driving license, how to help with medical assistance, or how seek employment. Im and Rosenberg [51] illustrate the diversity of topics that were delivered in their intervention and how this was conducted in a culturally responsive way:

"Key terms of the intervention topics, such as stress, acculturation, mental health, nutrition, healthy lifestyle, and community, were examined as a team in both languages 
and the peer facilitators had additional meetings prior to each session to go over the contents in Nepali".

This participant describes how learning in the peer group setting was used in order to support integration with the wider community. The group offered a safe space to try out new behaviours that would not be consistent with the participants culture within their country of origin. Thus, for this participant, the peer group helped support the acculturation process:

"In my culture, it is very hard to look at a person face-to-face [ . . ] and [ . . ] being women, you don't just laugh or smile [... I And when we practice sitting, facing one another, talking [ ... ] it changed my relationship because I practice it with other community, the wider community, looking at the person's eyes and smile". [52].

The following five articles reported on interventions that were beneficial for trauma and psychological distress during resettlement: [44-48]. Three of these studies exclusively focused on trans-theoretical scalable manualised interventions developed by the World Health Organisation (WHO) for psychological issues such as trauma, depression and anxiety [46-48]. Although these studies had small effect sizes, effects were not demonstrated at follow up in the Purgato et al. [47] study, and at three months the intervention effectiveness was greatly reduced in the Tol et al. [48] intervention. However, as both these studies were conducted in camps in host countries, the effectiveness may have been reduced due to contextual factors. Trans et al. [44] reported on reductions in depression, stress (including acculturation stress) perceived social support, and coping ability.

While Renner et al. [45] reported on reduced levels of anxiety, depression, and trauma symptoms, they note that wider resettlement issues were not successfully addressed, suggesting that in interventions resource capital may be essential during the resettlement process:

"Although all the participants had experienced positive changes in many aspects of their lives, there were many more issues that they had to cope with. At the end of the program the women were still confronted by bread-and-butter issues: 'can you tell me how to get a job?' (Wuail); 'do you have any friends who need housecleaning? I can do it' (Kui); 'there are seven of us living in my three-bedroom house. It is so small. I have to sleep in the kitchen (Nyamata)".

\section{Discussion}

The aim of this review was to identify and examine the body of peer-reviewed literature as it relates to the following research question: 'How does the literature describe interventions delivered by peers to refugees and asylum seekers during the resettlement process?' This scoping review included 14 peer-reviewed articles from an initial 639, with the characteristics of each study extracted and charted. The resulting data was used to report on two main themes. The studies included in this scoping review provide evidence for the effectiveness of culturally responsive peer-delivered interventions with diverse refugees and asylum seekers as they transition through the resettlement process in mainly high-income Western countries, with the exception of Uganda.

At the same time, peer support during the resettlement process seems to be a relatively untapped area in the extant literature. The research in this area is still in its infancy, and as such, much of the interventions in this review are localised and heterogeneous in terms of methodologies, training, content, and outcomes. Paradoxically, it may be that this heterogeneity is what makes these interventions successful, as they are localised not just to individual countries and contexts but to ethnic identities also. This would be consistent with the general cultural competency literature cited previously regarding the differential factors that can contribute to effective interventions with refugees [20] and the cultural competency literature in general [23-25]. Likewise, these finding seem to echo those found in Charles et al. [58] systematic review examining the importance of modifications to peer 
interventions, and the description provided by [26] earlier in this paper thar suggests that there may not be 'one model of best practice.'

On this note, the first, and one of the key findings from this scoping review, is the involvement of key stakeholders in co-producing peer interventions in terms of them being culturally responsive. Civil society organisations working with refugee populations, and refuges and asylum seekers themselves, played a large role in designing content that was culturally sensitive. In addition, such participatory approaches allowed for group membership and composition to consider further multicultural identities such as age, gender, and language [49-55]. This participation by the key stakeholders seems to be integral to successful interventions because they entail the added advantage of being naturally culturally responsive to the needs of each heterogeneous population, something which is very difficult to accomplish for the average practitioner attempting to move beyond a monocultural approach.

The next finding from this scoping review suggests that outcomes are vast and varied as they relate to the resettlement process. As such, in this review, peer interventions impacted on various social and wellbeing indicators across the wider ecological and social determinant systems previously identified as impacting on the resettlement process $[9,10,18]$. However, it should be noted that interventions that largely focus on psychopathology [45-48] even in the context of programmes with some focus on resettlement issues, failed to address many of these social determinants of health. However, there are small benefits from brief manualised interventions that can ameliorate various forms of non-diagnosable psychological distress.

The findings further support that resettlement was improved through enhanced community integration through acculturation and belonging [49-55] and while specific interventions such as connecting participants to community resources helped this process $[44,50,55]$ group members have their own knowledge capital that is used to support peer group members [52,53].

The final finding centres on training provided to peers, which is experienced as being integral. However, the optimal level of training remains unknown; again, this seems to be consistent with the general peer work literature [58]. While only three studies spoke to the impact of training on those delivering the interventions, the findings do suggest that effective interventions have a facilitative and communicative skills element to them [49-55] while also providing information on how to access community resources to support capital, acculturation, and community integration [44,49-53]. Training and supportive supervision provided to those delivering interventions is integral, as it can impact on peers building their sense of effectiveness, while a lack of training can leave peers feeling ill prepared to face challenging tasks in their roles and deliver protocols with fidelity.

Regarding the individual studies in this scoping review, the quality was heterogeneous in terms of methodologies. Four studies had a qualitative sample size of ten or under $[49,54-56]$ with six larger studies that included pre-post data [44-48,50]. Three studies reported on a mixed methods approach [46,49,52].while four of the studies did include a randomised control trial [45-48]. While appreciating the benefit of the RCT, I tend to share concerns noted by Bonnell et al. [59] regarding their appropriateness and the utility of this methodology in a sociological setting, especially where there are no intervention protocols. However, as all the RCTs and quantitative studies share many of the same outcome measures, a systematic review and meta-analysis should be considered.

\subsection{Limitations of this Scoping Review}

There are several limitations to this scoping review that should be considered when interpreting the findings. Firstly, it would be amiss not to acknowledge that this review was conducted by one person only, and while rigorous scoping review protocols and PRISMA guidelines were followed, it remains that an additional reviewer may have been beneficial with regards to analysis and searching. Secondly, the search strategy may have also been limited, in that four databases were only searched, and the keywords used may have 
provided for a narrow selection of peer-reviewed literature. It's possible, for example, that there are other sources of evidence where resettlement and peer interventions were reported on as secondary outcomes. Thirdly, this was not an exhaustive search as the grey literature was not included due to time constraints. Fourthly, the reliance on peerreviewed English articles means the review may have missed important studies conducted in different countries. Finally, while most studies included in this scoping review had participation from refugees and asylum seekers, this review could have benefited from participation by these key stakeholders. This could have been achieved by including the stakeholders in a sixth and optional stage of scoping reviews [38,60], however, the reviewer did not have the resources to make this a reality

\subsection{Implications for Practice, Policy, and Research}

Research of peer interventions during the resettlement process for refugees and asylum seekers is still in its infancy and heterogeneous in nature. However, based on the findings presented in this, the first scoping review of the literature, some tentative recommendations are provided. The evidence presented in this review suggests that various interventions delivered by peers are effective for addressing many of the resettlement challenges across the broader ecological system of needs of refugees and asylum seekers in various countries. This effectiveness is demonstrated through benefits in emotional wellbeing, acculturation, community integration, and better access to community resources. As such, practitioners and organisations delivering peer interventions should focus on addressing these key areas. While interventions focusing on psychopathology seem to be effective in ameliorating trauma and other forms of mental health difficulties, wider social determinants across the ecology are not addressed. It is also possible that interventions delivered to refugees and asylum seekers who are accommodated in camps are less effective as evidenced by the reduced effectiveness of psychological interventions at follow up. Thus, where peers and organisations are exclusively delivering mental health interventions to refugees and asylum seekers during resettlement, consideration should be given to case management interventions also.

At the same time, training is integral to the peer role, and initial findings presented here suggest training with a focus on communication skills and how peers can help refuges and asylum seekers access community resources is helpful. Additionally, interventions that offer structure through manualised protocols are also effective when supported by training regimes and supervision. Policy makers can use the findings from this review to inform strategies to support the wider integration process, with peers playing an important role through participating in the co-production of policy with regards to resettlement interventions. Furthermore, research with larger sample sizes needs to be conducted both to provide further evidence of peer's interventions but also regarding the level of training needed for peers to be effective. Participatory approaches to research are one of the main findings in this review, and the author encourages all further research to involve refugees and asylum seekers, and the civil society organisations that work with them, in the co-production of culturally responsive models.

Future research using theoretical frameworks such as the ecological model may prove beneficial for establishing an evidence base regarding the level of impact across the system that peer interventions can produce. While the political system is unlikely to be impacted, the interaction of peer interventions on the other levels of the system may provide useful findings for practice and policy. Finally, a systematic review and meta-analysis should be considered. Many of the outcome measures used in the quantitative studies are the same, thus pooling effect sizes may be possible.

\section{Conclusions}

This scoping review of 14 peer-reviewed studies demonstrates that peer support that is co-produced with refugees and asylum seekers, in conjunction with civil society organisations, is effective across the wider ecological system of needs this population of 
people have during resettlement. One of the key findings is centred on how participatory approaches to designing the content and processes of these interventions contribute to a culturally responsive intervention. While many of the interventions were heterogeneous in terms of their content, outcomes, and training, peers in these studies reported benefits in emotional support, community integration, acculturation, and better access to services. As for the training provided to peers to deliver these interventions, the optimal training regime is still unclear. However, facilitative interpersonal skills, how to deal with challenging dynamics, and training around how to support or refer refugees and asylum seekers to community resources seems to be beneficial, while several manualised interventions with scalability also demonstrate some effectiveness. At the same time, training or lack thereof can impact the peer's sense of effectiveness in their role in positive and negative ways, and supports are needed for peers to be effective. To the author's knowledge, this is the first scoping review on peer interventions used to support refugees and asylum seekers during resettlement and provides evidence of the effectiveness of such interventions. As such, this review contributes to the extant literature by demonstrating that interventions that are designed and adapted in conjunction with key stakeholders are naturally culturally responsive to heterogeneous and culturally diverse populations in mainly Western highincome societies.

Supplementary Materials: The following supporting information can be downloaded at: https: / / www.mdpi.com/article/10.3390/traumacare2010005/s1, Table S1: Charting Data Form.

Funding: No funding was received for this study.

Institutional Review Board Statement: Ethical review and approval were waived for this study as per advice from the NHS Health Re-search Authority (UK) decision tool, since it is a scoping review of published literature.

Informed Consent Statement: Participant consent was waived since no members of the public were involved in the design, conduct of this study, or reporting of this research.

Data Availability Statement: All data generated as part of this study are included in the article.

Conflicts of Interest: The author declares no conflict of interest.

\section{References}

1. World Migration Report (2020); International Organisation for Migration: Geneva, Switzerland, 2020; ISBN 978-92-9068-789-4.

2. UN High Commissioner for Refugees (UNHCR). Report of the United Nations High Commissioner for Refugees Covering the Period 1 July 2016-30 June 2017, 25 September 2017, A/72/12. Available online: https:/ /www.refworld.org/docid/59c8d9594. html (accessed on 23 February 2022).

3. UNHCR. Global Trends: Forced Displacement in 2019. 2020. Available online: https:/ /www.unhcr.org/5ee200e37.pdf (accessed on 8 October 2021).

4. Bustamante, L.; Cerqueira, R.O.; Leclerc, E.; Brietzke, E. Stress, trauma, and posttraumatic stress disorder in migrants: A comprehensive review. Rev. Bras. Psiquiatr. 2017, 40, 220-225. [CrossRef] [PubMed]

5. Bogic, M.; Njoku, A.; Priebe, S. Long-term mental health of war-refugees: A systematic literature review. BMC Int. Health Hum. Rights 2015, 15, 29. [CrossRef] [PubMed]

6. Henkelmann, J.R.; de Best, S.; Deckers, C.; Jensen, K.; Shahab, M.; Elzinga, B.; Molendijk, M. Anxiety, depression and posttraumatic stress disorder in refugees resettling in high-income countries: Systematic review and meta-analysis. BJPsych Open 2020, 6, e68. [CrossRef] [PubMed]

7. Morina, N.; Akhtar, A.; Barth, J.; Schnyder, U. Psychiatric disorders in refugees and internally displaced persons after forced displacement: A Systematic Review. Front. Psychiatry 2018, 9, 433. [CrossRef]

8. Steel, Z.; Chey, T.; Silove, D.; Marnane, C.; Bryant, R.A.; van Ommeren, M. Association of torture and other potentially traumatic events with mental health outcomes among populations exposed to mass conflict and displacement: A systematic review and meta-analysis. JAMA 2009, 302, 537-549. [CrossRef]

9. Blackmore, R.; Boyle, J.A.; Fazel, M.; Ranasinha, S.; Gray, K.M.; Fitzgerald, G.; Misso, M.; Gibson-Helm, M. The prevalence of mental illness in refugees and asylum seekers: A systematic review and meta-analysis. PLoS Med. 2020, 17, e1003337. [CrossRef]

10. Gleeson, C.; Frost, R.; Sherwood, L.; Shevlin, M.; Hyland, P.; Halpin, R. Post-migration factors and mental health outcomes in asylum-seeking and refugee populations: A systematic review. Eur. J. Psychotraumatol. 2020, 11, 1793567. [CrossRef] 
11. Van der Boor, C.F.; Amos, R.; Nevitt, S.; Dowrick, C.; White, R.G. Systematic review of factors associated with quality of life of asylum seekers and refugees in high-income countries. Confl. Health 2020, 14, 48. [CrossRef]

12. McGarry, O.; Hannigan, A.; Manuela De Almeida, M.; Severoni, S.; Puthoopparambil, S.J.; MacFarlane, A. What Strategies to Address Communication Barriers for Refugees and Migrants in Health Care Settings Have Been Implemented and Evaluated across the WHO European Region? WHO Regional Office for Europe: Copenhagen, Denmark, 2008.

13. Turrini, G.; Purgato, M.; Acarturk, C.; Anttila, M.; Au, T.; Ballette, F.; Bird, M.; Carswell, K.; Churchill, R.; Cuijpers, P.; et al. Efficacy and acceptability of psychosocial interventions in asylum seekers and refugees: Systematic review and meta-analysis. Epidemiol. Psychiatr. Sci. 2019, 28, 376-388. [CrossRef]

14. Çetrez, Ö.A.; DeMarinis, V.; Sundvall, M.; Fernandez-Gonzalez, M.; Borisova, L.; Titelman, D. A Public Mental Health Study among Iraqi Refugees in Sweden: Social Determinants, Resilience, Gender, and Cultural Context. Front. Sociol. 2021, 26, 6:551105. [CrossRef]

15. Goodkind, J.R.; Bybee, D.; Hess, J.M.; Amer, S.; Ndayisenga, M.; Greene, R.N.; Choe, R.; Isakson, B.; Baca, B.; Pannah, M. Randomized Controlled Trial of a Multilevel Intervention to Address Social Determinants of Refugee Mental Health. Am. J. Community Psychol. 2020, 65, 272-289. [CrossRef] [PubMed]

16. Goodkind, J.R. Effectiveness of a Community-Based Advocacy and Learning Program for Hmong Refugees. Am. J. Community Psychol. 2006, 36, 387-408. [CrossRef] [PubMed]

17. Kim, W.; Kim, I.; Lin, L.; Baltimore, K.; Lin, L. Social Determinants of Mental Health Among Karen Refugees from Burma. Community Ment. Health J. 2021, 1-12. [CrossRef] [PubMed]

18. Badali, J.J.; Grande, S.; Mardikian, K. From passive recipient to community advocate: Reflections on peer-based resettlement programs for Arabic-speaking refugees in Canada. Glob. J. Community Psychol. Pract. 2017, 8, 1-31.

19. Huey, S.J., Jr.; Tilley, J.L.; Jones, E.O.; Smith, C.A. The contribution of cultural competence to evidence-based care for ethnically diverse populations. Annu. Rev. Clin. Psychol. 2014, 10, 305-338. [CrossRef]

20. Lau, L.S.; Rodgers, G. Cultural Competence in Refugee Service Settings: A Scoping Review. Health Equity 2021, 5, 124-134 [CrossRef]

21. Perera, C.; Salamanca-Sanabria, A.; Caballero-Bernal, J.; Feldman, L.; Hansen, M.; Bird, M.; Hansen, P.; Dinesen, C.; Wiedemann, N.; Vallières, F. No implementation without cultural adaptation: A process for culturally adapting low-intensity psychological interventions in humanitarian settings. J. Confl. Health 2020, 14, 46. [CrossRef]

22. Riza, E.; Kalkman, S.; Coritsidis, A.; Koubardas, S.; Vassiliu, S.; Lazarou, D.; Karnaki, P.; Zota, D.; Kantzanou, M.; Psaltopoulou, T.; et al. Community-Based Healthcare for Migrants and Refugees: A Scoping Literature Review of Best Practices. Healthcare 2020, 8, 115. [CrossRef]

23. Benish, S.G.; Quintana, S.; Wampold, B.E. Culturally adapted psychotherapy and the legitimacy of myth: A direct-comparison meta-analysis. J. Couns. Psychol. 2011, 58, 279-289. [CrossRef]

24. Griner, D.; Smith, T.B. Culturally adapted mental health intervention: A meta-analytic review. J. Psychother. 2006, 43, 531-548. [CrossRef]

25. Smith, T.B.; Rodríguez, M.D.; Bernal, G. Culture. J. Clin. Psychol. 2011, 67, 166-175. [CrossRef] [PubMed]

26. Riggs, E.; Davis, E.; Gibbs, L.; Block, K.; Szwarc, J.; Casey, S.; Duell-Piening, P.; Waters, E. Accessing maternal and child health services in Melbourne, Australia: Reflections from refugee families and service providers. BMC Health Serv. Res. $2012,12,117$. [CrossRef] [PubMed]

27. Bellamy, C.; Schmutte, T.; Davidson, L. An update on the growing evidence base for peer support. Ment. Health Soc. Incl. 2017, 21, 161-167. [CrossRef]

28. Fuhr, D.C.; Salisbury, T.T.; De Silva, M.J.; Atif, N.; van Ginneken, N.; Rahman, A.; Patel, V. Effectiveness of peer-delivered interventions for severe mental illness and depression on clinical and psychosocial outcomes: A systematic review and metaanalysis. Soc. Psychiatry Psychiatr. Epidemiol. 2014, 49, 1691-1702. [CrossRef] [PubMed]

29. King, A.J.; Simmons, M.B. A systematic review of the attributes and outcomes of peer work and guidelines for reporting studies of peer interventions. Psychiatr. Serv. 2018, 69, 961-977. [CrossRef]

30. Lloyd-Evans, B.; Mayo-Wilson, E.; Harrison, B.; Istead, H.; Brown, E.; Pilling, S.; Johnson, S.; Kendall, T. A systematic review and meta-analysis of randomised controlled trials of peer support for people with severe mental illness. BMC Psychiatry 2014, 14, 39. [CrossRef]

31. White, S.; Foster, R.; Marks, J.; Morshead, R.; Goldsmith, L.; Barlow, S.; Sin, J.; Gillard, S. The effectiveness of one-to-one peer support in mental health services: A systematic review and meta-analysis. BMC Psychiatry 2020, 20, 534. [CrossRef]

32. Shaw, S.A.; Funk, M. A Systematic Review of Social Service Programs Serving Refugees. Res. Soc. Work Pract. 2019, $29,847-862$. [CrossRef]

33. Burns, R.; Zhang, C.X.; Patel, P.; Eley, I.; Campos-Matos, I.; Aldridge, R.W. Migration health research in the United Kingdom: A scoping review. J. Migr. Health 2021, 4, 100061. [CrossRef]

34. Villarroel, N.; Hannigan, A.; Severoni, S. Migrant health research in the Republic of Ireland: A scoping review. BMC Public Health 2019, 19, 324. [CrossRef]

35. Essex, R.; Kalocsányiová, E.; Rumyantseva, N.; Jameson, J. Trust Amongst Refugees in Resettlement Settings: A Systematic Scoping Review and Thematic Analysis of the Literature. Int. Migr. Integr. 2021, 1-26. [CrossRef] 
36. Fennig, M.; Denov, M. Interpreters working. in mental health settings with refugees: An interdisciplinary scoping review. Am. J. Orthopsychiatry 2021, 91, 50-65. [CrossRef] [PubMed]

37. Wachter, K.; Bunn, M.; Schuster, R.C.; Boateng, O.G.; Cameli, K.; Johnson-Agbakwu, C. A scoping review of social support research among refugees in resettlement: Implications for conceptual and empirical research. J. Refug. Stud. 2021, 34, feab040. [CrossRef]

38. Page, M.J.; McKenzie, J.E.; Bossuyt, P.M.; Boutron, I.; Hoffmann, T.C.; Mulrow, C.D.; Shamseer, L.; Tetzlaff, J.M.; Akl, E.A.; Brennan, S.E.; et al. The PRISMA 2020 statement: An updated guideline for reporting systematic reviews. BMJ 2021, 372, n71. [CrossRef] [PubMed]

39. Arksey, H.; O'Malley, L. Scoping studies: Towards a methodological framework. Int. J. Soc. Res. Methodol. 2005, 8, 19-32. [CrossRef]

40. Mays, N.; Roberts, E.; Popay, J. Synthesising research evidence. In Studying the Organisation and Delivery of Health Services: Research Methods; Fulop, N., Allen, P., Clarke, A., Black, N., Eds.; Routledge: London, UK, 2001; pp. 188-220.

41. Tricco, A.C.; Lillie, E.; Zarin, W. A scoping review on the conduct and reporting of scoping reviews. BMC Med. Res. Methodol. 2016, 16, 15. [CrossRef]

42. Methley, A.M.; Campbell, S.; Chew-Graham, C.; McNally, R.; Cheraghi-Sohi, S. PICO, PICOS and SPIDER: A comparison study of specificity and sensitivity in three search tools for qualitative systematic reviews. BMC Health Serv. Res. 2014, 14, 579. [CrossRef]

43. Braun, V.; Clarke, V. Using thematic analysis in psychology. Qual. Res. Psychol. 2006, 3, 77-101. [CrossRef]

44. Tran, A.N.; Ornelas, I.J.; Kim, M.; Perez, G.; Green, M.; Lyn, M.J.; Corbie-Smith, G. Results from a pilot promotora program to reduce depression and stress among immigrant Latinas. Health Promot. Psychol. 2014, 15, 365-372. [CrossRef]

45. Renner, W.; BÃanninger-Huber, E.; Peltzer, K. Culture-sensitive and resource-oriented peer (CROP)-groups as a community based intervention for trauma survivors: A randomized controlled pilot study with refugees and asylum seekers from chechnya. Australas. J. Disaster Trauma Stud. 2011, 1, 1-13.

46. De Graaff, A.M.; Cuijpers, P.; Acarturk, C.; Bryant, R.; Burchert, S.; Fuhr, D.C.; Huizink, A.C.; de Jong, J.; Kieft, B.; Knaevelsrud, C.; et al. Effectiveness of a peer-refugee delivered psychological intervention to reduce psychological distress among adult Syrian refugees in the Netherlands: Study protocol. Eur. J. Psychotraumatol. 2020, 20, 1694347. [CrossRef] [PubMed]

47. Purgato, M.; Carswell, K.; Tedeschi, F.; Acarturk, C.; Anttila, M.; Au, T.; Bajbouj, M.; Baumgartner, J.; Biondi, M.; Churchill, R.; et al. Effectiveness of Self-Help Plus in preventing mental disorders in refugees and asylum seekers in Western Europe: A multinational randomized controlled trial. Psychother. Psychosom. 2021, 90, 403-414. [CrossRef] [PubMed]

48. Tol, W.A.; Leku, M.R.; Lakin, D.P.; Carswell, K.; Augustinavicius, J.; Adaku, A.; Au, T.M.; Brown, F.L.; Bryant, R.A.; GarciaMoreno, C.; et al. Guided self-help to reduce psychological distress in South Sudanese female refugees in Uganda: A cluster randomised trial. Lancet Glob. Health 2020, 8, e254-e263. [CrossRef]

49. Abrahamson, A.; Andersson, J.; Springett, J. Building bridges or negotiating tensions? Experiences from a project aimed at enabling migrant access to health and social care in Sweden. Divers. Health Care 2009, 6, 85-95.

50. Block, A.M.; Aizenman, L.; Saad, A.; Harrison, S.; Sloan, A.; Vecchio, S.; Wilson, V. Peer support groups: Evaluating a Culturally Grounded, STRENGTHS-BASED approach for work with refugees. Adv. Soc. Work. 2018, 18, 930-948. [CrossRef]

51. Im, H.; Rosenberg, R. Building Social Capital through a Peer-Led Community HealthWorkshop: A Pilot with the Bhutanese Refugee Community. J. Community Health 2016, 41, 509-517. [CrossRef]

52. Koh, L.C.; Walker, R.; Wollersheim, D.; Liamputtong, P. I think someone is walking with me: The use of mobile phone for social capital development among women in four refugee communities. Int. J. Migr. Health Soc. Care 2018, 14, 411-424. [CrossRef]

53. Stewart, M.; Simich, L.; Shizha, E.; Makumbe, K.; Makwarimba, E. Supporting African refugees in Canada: Insights from a support intervention. Health Soc. Care Community 2012, 20, 516-527. [CrossRef]

54. Wollersheim, D.; Koh, L.; Walker, R.; Liamputtong, P. Constant connections: Piloting a mobile phone-based peer support program for Nuer (southern Sudanese) women. Aust. J. Prim. Health 2013, 19, 7-13. [CrossRef]

55. Paloma, V.; de la Morena, I.; Sladkova, J.; López-Torres, C.A. peer support and peer mentoring approach to enhancing resilience and empowerment among refugees settled in southern Spain. J. Community Psychol. 2020, 48, 1438-1451. [CrossRef]

56. Shaw, A.S. Bridge Builders: A Qualitative Study Exploring the Experiences of Former Refugees Working as Caseworkers in the United States. J. Soc. Serv. Res. 2014, 40, 284-296. [CrossRef]

57. Paloma, V.; de la Morena, I.; Lopez Torres, C. Promoting posttraumatic growth among the refugee population in Spain: A community-based pilot intervention. Health Soc. Care Community 2020, 28, 127-136. [CrossRef] [PubMed]

58. Charles, A.; Thompson, D.; Nixdorf, R.; Ryan, G.; Shamba, D.; Kalha, J.; Moran, G.; Hiltensperger, R.; Mahlke, C.; Puschner, B.; et al. Typology of modifications to peer support work for adults with mental health problems: Systematic review. Br. J. Psychiatry 2020, 216, 301-307. [CrossRef] [PubMed]

59. Bonell, C.; Allen, E.; Warren, E.; McGowan, J.; Bevilacqua, L.; Jamal, F.; Legood, R.; Wiggins, M.; Opondo, C.; Mathiot, A.; et al. Effects of the Learning Together intervention on bullying and aggression in English secondary schools (INCLUSIVE): A cluster randomised controlled trial. Lance 2018, 392, 2452-2464. [CrossRef]

60. Levac, D.; Colquhoun, H.; O’Brien, K.K. Scoping studies: Advancing the methodology. Implement. Sci. 2010, 5, 69. [CrossRef] 